\title{
Terrain Backscatter and Oil Sand Exploration: Average Reflectivity Analysis
}

\author{
Maurice Ezeoke ${ }^{I}$, Cristina Borda Fortuny, Kenneth Tong \\ Department of Electronic and Electrical Engineering \\ University College London \\ London, England \\ uceemse@ucl.ac.uk
}

\begin{abstract}
A barefaced terrain scattering model for oil sand exploration will enable the discrimination of different terrain types using incoherent polarimetric decomposition. We use computer electromagnetic modeling with finite integration techniques and a network analyzer based indoor polarimetric scatterometer to determine and analyze terrain backscatter. The terrain targets vary in dielectric, physical and chemical properties so we investigated their intrinsic material effects on electromagnetic reflectivity prior to obtaining the average normalized radar cross section. We obtain a strong correlation between the models and empirical measurement for co-polar $(\mathrm{HH}$ and VV) polarizations at different incident geometry and frequency $L-, C$ - and $X$-band.
\end{abstract}

Keywords-average terrain reflectivity; backscatter measurement; computer electromagnetic model; laboratory scatterometersystem; oil sand exploration

\section{INTRODUCTION}

Although there has been frequently recurring interest in the use of synthetic aperture radar (SAR) and polarimetry for remote sensing, practical application to conventional hydrocarbon exploration has been hindered by the penetration depth, $\delta_{p}$ of electromagnetic (EM) energy. High oil prices and surficial expression of unconventional petroleum reservoirs such as oil sands and shale rock has revived interest in radar for petroleum exploration of oil sands. Oil sands are mainly a heterogeneous mixture of bitumen, sand and water. A key requirement for radar remote sensing is to extract geoscientific information after microwave scattering from terrain [1], [2].

Here we consider a method to obtain the backscattering coefficient from oil sand in relation to intrinsic terrain properties. This is a precursor to developing a barefaced terrain model with important implications for discriminating between low backscatter environments with similar geography and resultant clutter. The developed technique will also aid terrain classification in the absence of access to satellite or airborne SAR calibration imagery over specific areas of interest while the average reflectivity results may further serve as training data for supervised decomposition methods [3].

Distributed targets present unique imaging problems for radar remote sensing due to the presence of multiplicative speckle noise. Unlike coherent point targets which are easier discriminated from surrounding objects due to discernible physical features, distributed targets are best determined from the average or dominant scattering mechanism [3], [4]. Target information is described by the radar cross section (RCS) or scattering coefficient, $\sigma$ for point targets and average normalized RCS (nRCS) per unit area or backscattering coefficient $\sigma^{0}$ for distributed targets. Both depend on target geometric and dielectric properties as well as sensor parameters like incident geometry, polarization and spatial resolution. Therefore $\sigma^{0}$ is characterized for specific frequency, $f$ and polarization of incident $\left(\theta_{i},{ }_{i}\right)$ and scattered $\left(\theta_{s},{ }_{s}\right)$ wave directions. Experiments have been carried out to determine scattering relationships for farmlands, desert and vegetated surfaces using airborne or satellite scatterometers but rather little information has been published on radar performance for oil sand exploration [5]-[7]. We configured an indoor measurement system to act as a radar scatterometer.

The interpretation of SAR imagery is non-linear, varying on a gray scale palette where low backscattered signals are dark and high backscattered signals are merely brighter. Hence it is customary to first model the EM wave interaction with the target or scattering systems as shown in Fig. 1. Here an incident polarized wave, $\mathbf{E}^{i}$ interacts with the distributed scatterer $[M]$ through combination of wave propagation, attenuation and scattering. In [4], the classical Stokes vector of a wave has been solved for an intensity vector, $\mathbf{k}$ to give a Mueller matrix relationship for incident and scattered waves directions with $[M]$ as (Fig.1 and (1)):

$$
\mathbf{k}_{s}=[M] \mathbf{k}_{i}
$$

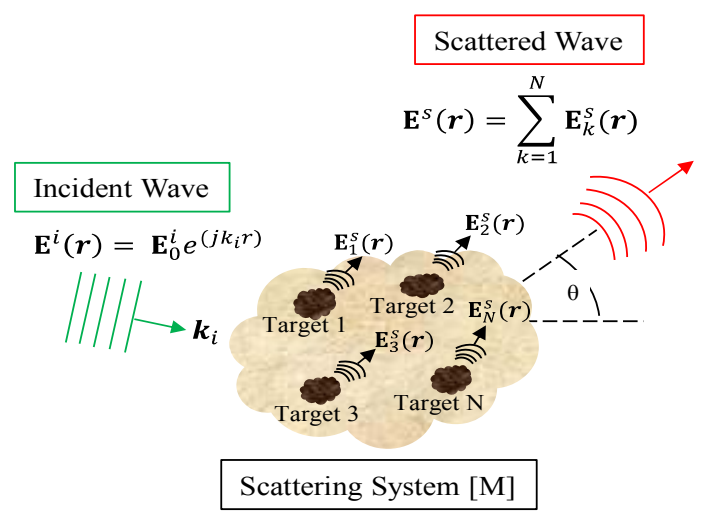

Fig. 1. General scattering geometry showing interaction of EM wave and extended target

\footnotetext{
${ }^{1}$ Petroleum Technology Development Fund and National Space Research
} and Development Agency 
In the scattering plane, $\theta_{S}$ is the scattering angle where $\theta_{S}=$ $0^{\circ}$ is forward scatter and $\theta_{S}=180^{\circ}$ is backscattered. For terrain the resulting scattered field, $\mathbf{E}^{s}$ is due to a coherent addition of scattered waves $\mathbf{E}_{k}^{s}(k=1,2 \ldots N)$ from independent targets that model the extended target scatterer. Therefore the incident and scattered EM waves, $\mathbf{E}^{i}$ and $\mathbf{E}^{s}$ at a distance $\mathbf{r}$ can be represented by a Jones vector (2) and (3):

$$
\begin{aligned}
\mathbf{E}^{i}(\mathbf{r}) & =\mathbf{E}_{0}^{i} e^{\left(j k_{i} r\right)} \\
\mathbf{E}^{s}(\mathbf{r}) & =\sum_{k=1}^{N} \mathbf{E}_{k}^{S}(\mathbf{r})
\end{aligned}
$$

Detailed mathematical formulation and decomposition of target vectors for converting $[M]$ to scattering matrix $[S]$ within backscatter problems is given in [3], [4] and [8]. The measured scattering or $S$-matrix $[S]$ corresponds to a complex target. The technique used to model, measure and analyze $\sigma^{0}$ from $[S]$ for diverse barefaced terrain with different properties is the purpose of this paper.

We develop a method to identify the average reflectivity of terrain in order to distinguish oil sand using 3D EM simulation and microwave measurement techniques. It will present new information on monostatic $\sigma_{M}^{0}$ for oil sand. Samples of the barefaced terrain investigated are shown in Fig. 2. The material under test (MUT) A - F represent beach sand, loamy farm soil (LFS), 10mm pebbles, 40mm gravel, hard oil sand (HOS) and viscous oil sand (VOS) respectively. Previously we determined the geochemical signature of the terrain using spectroscopy and also experimentally measured the dielectric permittivity ( $\varepsilon^{\prime}$ and $\left.\varepsilon^{\prime \prime}\right)$ of MUT A, B, E and F using a vector network analyzer (VNA) and dielectric probe kit.

Detailed measurement results for real and imaginary permittivity, $\varepsilon^{\prime}$ and $\varepsilon^{\prime \prime}$ are at [9], [10]. We use our approach developed in [11] for the 3D EM simulation system. In the next section we introduce both the radar system model and measurement system. Thereafter we describe the reflectivity calculation in section 3 before result analysis in section 4 . We conclude in section 5 .

\section{RADAR SYSTEM MODEL}

\section{A. Finite Integral Technique}

The aim of 3D EM simulation is to solve the integral equations which represent the interaction of EM waves with an object. Several solvers may be used to solve for the scattered field, $\mathbf{E}^{s}$ reflected from an object. They include full wave solvers such as the method of moments or approximate methods such as finite element methods or finite integration technique (FIT). We represent the general scattering geometry from Fig.1 using FIT. The FIT was implemented with the computer simulation technology microwave studio (CST MWS) commercial software because it is adaptable, easy to use and provides a good approximation of the solution [12].

The FIT discretization scheme implemented by CST involves a decomposition of the computational domain, $C_{D}$ in to a finite number of smaller mesh cells $C_{d}$ [13]. This means that every aspect of the simulation is represented by $3 \mathrm{D}$ mesh cells located in two orthogonal grids. A primary grid $\mathbf{G}$ contains the mesh cells while another orthogonal grid mesh $\widetilde{\mathbf{G}}$ is set up orthogonally to $\mathbf{G}$. The spatial discretization permits flexibility in modelling the terrain attributes such as surface roughness and inclusion of material dielectric properties.

The Maxwell constitutive equations that characterize the properties of terrain in terms of permittivity $\mathcal{E}^{\prime}$, permeability $\mu$ and conductivity $\sigma$ are given by (4):

$$
\mathbf{D}=\varepsilon^{\prime} \mathbf{E} ; \quad \mathbf{B}=\mu \boldsymbol{H} ; \quad \mathbf{J}=\sigma \mathbf{E} ;
$$

Here $\mathbf{D}(\mathbf{r}, t), \mathbf{B}(\mathbf{r}, t)$ and $\mathbf{J}(\mathbf{r}, t)$ are the electric displacement, magnetic flux density and current present at the space-time $(\mathbf{r}, t)$ being considered. The constitutive equations from (4) are represented through FIT by (5)

$$
\mathbf{D}=\boldsymbol{d}_{\varepsilon^{\prime}} \mathbf{E} ; \quad \mathbf{B}=\boldsymbol{d}_{\mu} \boldsymbol{H} ; \quad \mathbf{J}=\boldsymbol{d}_{\sigma} \mathrm{E} ;
$$

In this way $\boldsymbol{d}_{\varepsilon}, \boldsymbol{d}_{\mu}$ and $\boldsymbol{d}_{\sigma}$ represent the permittivity, permeability and conductivity matrices respectively. This gives us the opportunity to incorporate a matrix of the permittivity

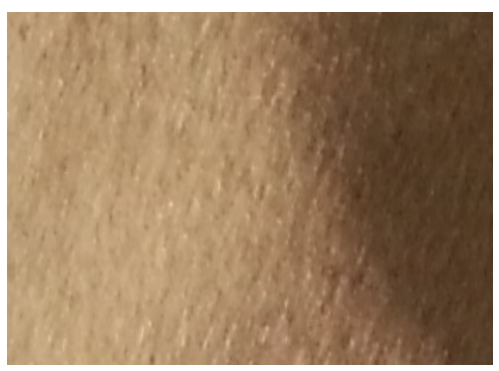

(MUT-A)

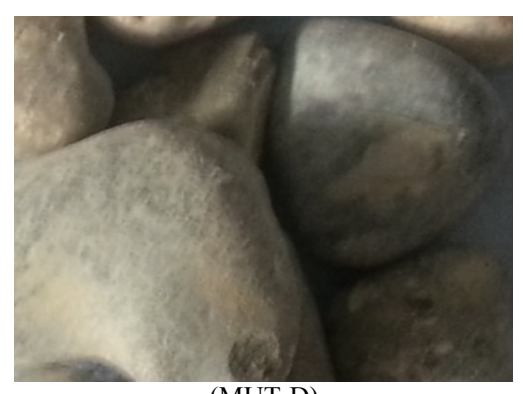

(MUT-D)

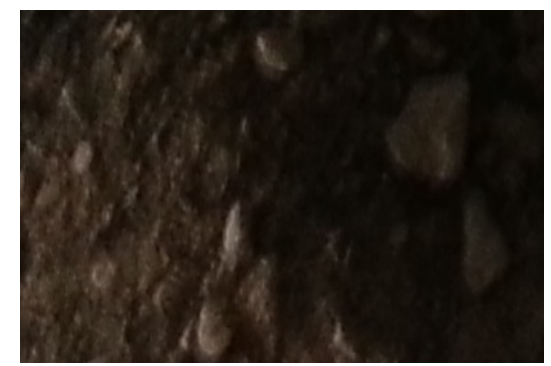

(MUT-B)

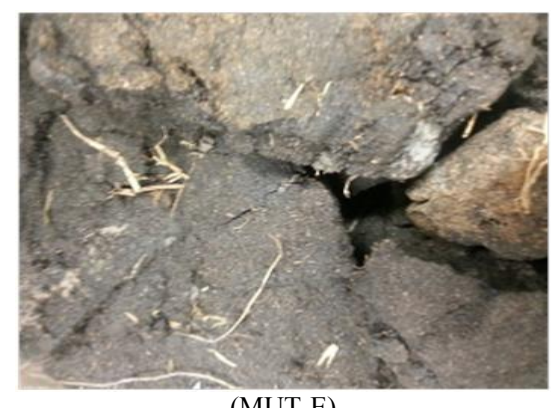

(MUT-E)

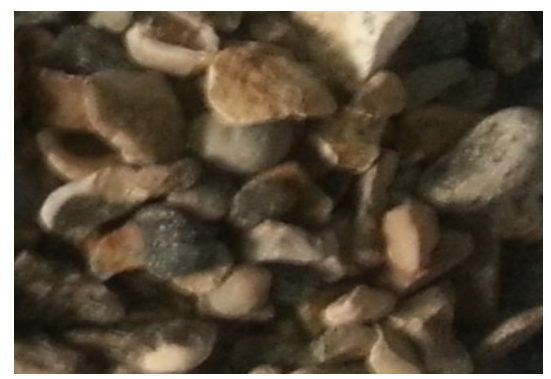

(MUT-C)

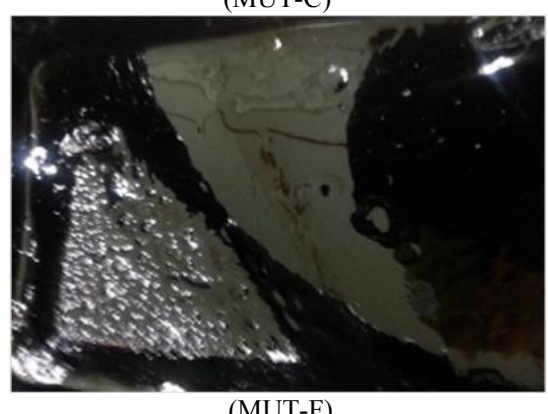

(MUT-F)

Fig. 2. Barefaced Terrain. First row: MUT: (A) Beach Sand (B) Loamy Farm Soil (C) 10mm pebbles. Second row: MUT: (D) 40mm Gravel (E) Hard Oil Sand (F) Viscous Oil Sand 
results as input into the terrain models with FIT. Dielectric permittivity of MUT-A and MUT-B with $10 \%$ and $20 \%$ weight percentage (wt. \%) water content respectively was measured.

The aim is to approximate the scattered field, $\mathbf{E}^{s}$ due to attributes of the scattering system, $[M]$. This is achieved by a transmitter dependent diagonal scattering tensor within the grid complexes $\mathbf{G}$ and $\widetilde{\mathbf{G}}$ enabling us to build the 3D EM model to accurately represent the measuring system. For the transmitterdependent diagonal scattering tensor, $\Gamma\left(\mathbf{r}, \mathbf{r}_{\mathrm{T}}\right)=\operatorname{diag}\left[\psi_{x}, \psi_{y}, \psi_{z}\right]$ the scattered field for a point within the computational domain $\left(C_{D}\right)$ is given by:

$$
\mathbf{E}^{S}\left(\mathbf{r}, \mathbf{r}_{T}\right) \approx \Gamma\left(\mathbf{r}, \mathbf{r}_{T}\right) \cdot \mathbf{E}^{i}\left(\mathbf{r}, \mathbf{r}_{T}\right) \quad \mathbf{r} \quad C_{D}
$$

The terrain scattering system and monostatic radar configuration was modeled using CST MWS [12].

\section{B. Polarimetric Radar Model}

The radar system model represented a monostatic radar system where the same antenna is used as both transmitter and receiver. In this case the area-extensive form of the radar equation describes the interaction of $\mathbf{E}^{i}$ with $[M]$ given by [1]:

$$
\bar{P}_{r}=\frac{\lambda^{2}}{(4 \pi)^{3}} \cdot \iint_{\begin{array}{c}
\text { Illuminated } \\
\text { Area }
\end{array}} \frac{P_{t} G_{t} G_{r} \sigma^{0} d A}{r^{4}}
$$

Here $\bar{P}_{r}$ is the average received power by an antenna with gain $G_{r}$ while $P_{t}$ is the power transmitted by an antenna with gain $G_{t}$. Also $d A$ is the illuminated elemental area that represents the scattering system at a distance $r$ from the transmitter. For our model we consider an elemental volume $d V(=d A d z)$ to represent the terrain features in 3D.

An antenna operating over the frequency range, $f$ from 0.8 10.5 $\mathrm{GHz}$ was designed then created in CST MWS. The antenna includes a tapered double ridged wave-guide to increase the bandwidth compared to the regular horn antenna [14]. This way we used the same antenna for multifrequency analysis at $f=1,7$ and $10 \mathrm{GHz}$. The main characteristics of the radar model and the laboratory scatterometer system (LSS) used in an anechoic chamber are presented in Table I.

TABLE I. SUMMARY OF RADAR CHARACTERISTICS FOR

\begin{tabular}{|c|c|c|}
\hline Property & Radar Model & LSS \\
\hline Receiver gain ${ }^{\mathrm{a}}$ & 7.5-14.3 dBi & $6.25-14.5 \mathrm{dBi}$ \\
\hline Transmitter gain & 7.5-14.3 dBi & $7-15 \mathrm{dBi}$ \\
\hline Beamwidth $^{\mathrm{b}}(3 \mathrm{~dB}) \mathrm{E} \times \mathrm{H}$ & $\begin{array}{l}87.8-15^{\circ} \mathrm{x} \\
60.9-30.3^{\circ} \\
\end{array}$ & $\begin{array}{c}100-18^{\circ} \times 63- \\
35^{\circ}\end{array}$ \\
\hline $\operatorname{Losses}^{c}$ & Nil & $0.5 \mathrm{~dB}$ \\
\hline Output power & $0 \mathrm{~dB}$ & $0 \mathrm{dBm}$ \\
\hline Transmitted Waveforms & Gaussian & Trace stimulus \\
\hline
\end{tabular}
SCATTEROMETER MODEL AND MEASUREMENT SYSTEM

Although connectors used were approximated with simple structures the dielectric material components were not ignored unlike in [15]. This increases accuracy at the expense of simulation time. The radiation pattern of the transmit antenna in terms of $\mathrm{E}$ and $\mathrm{H}$ planes is shown in Fig. 3. For the linearly polarized antenna the electric or E-field determines the antenna polarization. For horizontal $(\mathrm{H})$ polarization the E-field coincides with the azimuth $(A z$.) plane and the magnetic or $\mathrm{H}$ field coincides with the elevation $(E l$.) plane.

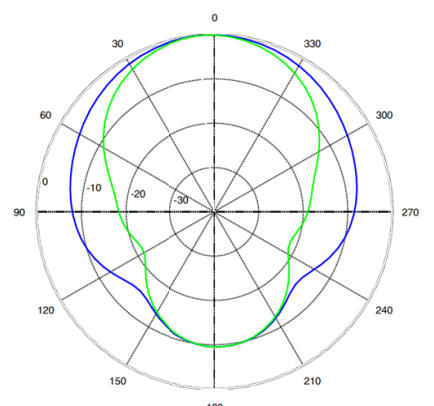

(a)

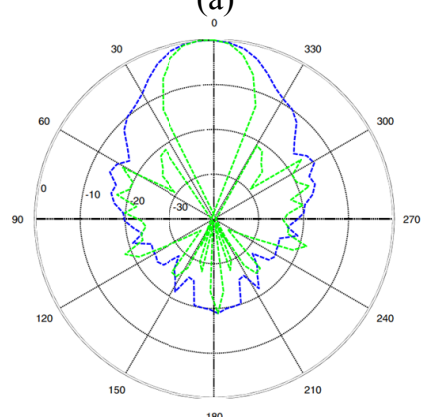

(b)

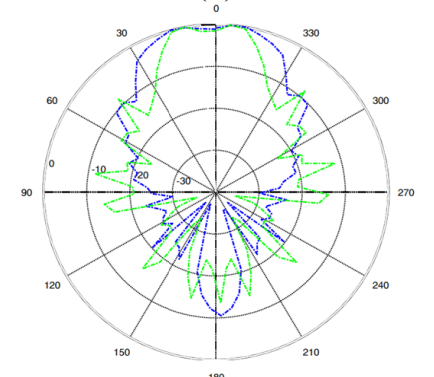

(c)

Fig. 3. Modelled radiation pattern of transmit antenna at $1 \mathrm{GHz}, 7 \mathrm{GHz}$ and $10 \mathrm{GHz}$. (a) Co-polarised E-field at $1 \mathrm{GHz}$ : solid blue line and co-polarised H-field at $1 \mathrm{GHz}$ : solid green line, (b) Co-polarised E-field at 7GHz: dashed blue line and co-polarised $\mathrm{H}$-field at 7GHz: dashed green line, (c) Co-polarised E-field at 10GHz: dotted blue blue line and co-polarised $\mathrm{H}$-field at 10GHz: dotted green line.

The terrain models were developed using an approach detailed in [11] in order to account for surface roughness variations. The simulation determined the monostatic reflectivity (S11) for the antenna and the scattered power for a representative plane wave using the configuration shown in Fig. 4. E-field probes were placed on the antenna surface, halfway to the terrain and on the terrain surface to observe the behaviour of the EM field in the $x, y$ and $z$ coordinates.

\section{Laboratory Scatterometer System}

The polarimetric radar system was set up in the University College London anechoic chamber. This LSS consisted of a 
Satimo SH800 wide band horn antenna, Hewlett-Packard 85052D calibration system, Rohde \& Schwarz ZNB40 VNA, Styrofoam polystyrene foam box (SPFB) and Meade Instruments LX80 tripod telescopic mounts arranged as shown in Fig. 4. The use of a VNA as intermediate frequency (IF) processor for the scatterometer reduced the hardware configuration complexity to consideration of RF components and imaging geometry as noted in [16]. Short, open, load and through (SOLT) calibration was performed before each measurement and the generated signal is transmitted through the SH800 antenna in $V$ or $H$ polarization to the target.

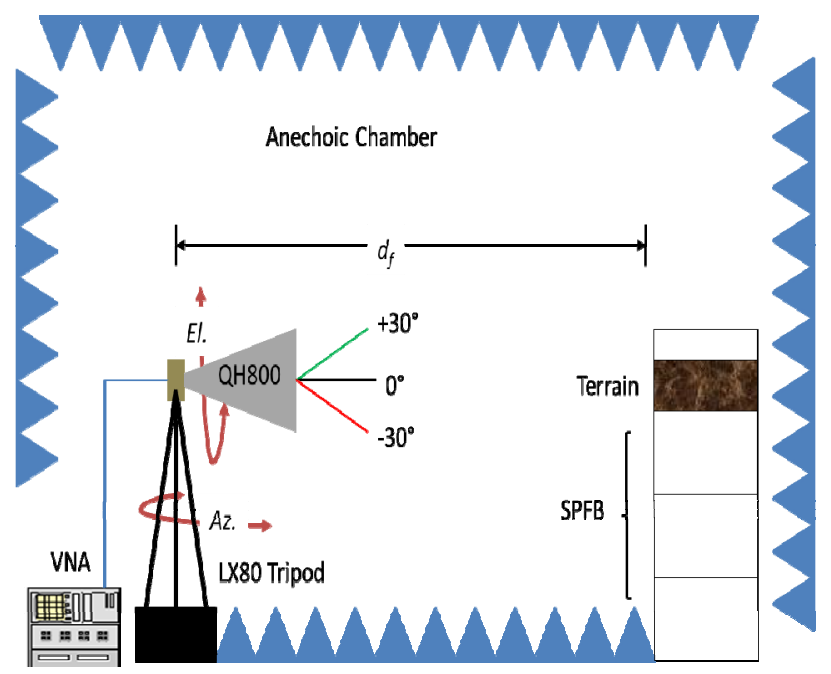

Fig. 4. Empirical laboratory scatterometer measurement system set up for multi-frequency terrain backscatter measurements at different incident geometry.

The SH800 ultrawideband horn antenna can operate over the frequency range $0.8-12 \mathrm{GHz}$. It was used both as transmitter and receiver for the LSS such that the pattern and gain of both the modeled and used antennas was similar (see Fig. 3). Characteristics of the SH800 antenna and VNA relevant to this work are given in Table I. In radar remote sensing, the imagery of terrain acquired by a SAR sensor borne on a satellite or airplane will be in the far field. The far field distance, $d_{\mathrm{F}}$ is related to the wavelength, $\lambda$ of the EM field being transmitted by an antenna of dimension $D$ according to:

$$
d_{F}=\frac{2 D^{2}}{\lambda}
$$

The limited size of a typical university anechoic chamber could hinder indoor measurements at low frequencies. However the use of a wideband antenna with fixed dimensions meant that we could still carry out far field measurements within the chamber. Terrain samples were placed in SPFB boxes at a distance, $r$ of $4.9 \mathrm{~m}$ which is greater than $10 \lambda$ away in free space. The SPFB boxes with measured dielectric permittivity, $\varepsilon_{\mathrm{r}}=1.03$ were used both as support stand and holding container. Terrain occupied $0.42 \mathrm{~m} \times 0.32 \mathrm{~m} \times 0.1 \mathrm{~m}$ due to the internal dimensions of the SPFB, so $d V=0.0134 \mathrm{~m}^{3}$.

\section{DETERMINATION OF TERRAIN REFLECTIVITY}

The response of the LSS without a target was stored and subtracted from the response with the target present in order to isolate the returns from the terrain target alone. For each measurement 5 - 10 data traces were continuously averaged before results were taken in order to improve the signal to noise ratio (SNR) as would obtain in a conventional satellite or airborne radar system.

For this approach we consider the scattering system $[M]$ of the area extensive target to be composed of a collection of statistically identical targets (see Fig. 1). Therefore the radar equation (7) can be rewritten to separate the effects of the target extent where $d P_{r}$ and $d \sigma$ are the average quantities obtained by the measurement system for the differential volume $d V$. From [1] we modify this to be:

$$
d P_{r}=\frac{P_{t} G_{t} G_{r} \lambda^{2}}{(4 \pi)^{3} r^{4}} \sigma^{0} d V .
$$

The total power received from the extended target requires integration over the illuminated area $A_{0}$.

$$
P_{r}=\iint_{A_{0}} \frac{P_{t} G_{t} G_{r} \lambda^{2}}{(4 \pi)^{3} r^{4}} \sigma^{0} d V .
$$

Hence the scattering coefficient, $\sigma^{0}$ is determined by the ratio of the average scattered power density to the average incident power density:

$$
\sigma^{0}=\frac{\langle\sigma\rangle}{A_{0}}=\frac{4 \pi r^{2}\left\langle\left|\mathbf{E}^{s}\right|^{2}\right\rangle}{\left|\mathbf{E}^{i}\right|^{2}} .
$$

A conventional radar scatterometer measures radar return as a function of range, $r$ with returns isolated for each range using time gating [17]. With the LSS we measured the backscattered power as a function of the different terrain type placed in the SPFB boxes at a specific distance, $r$ and rather than time-gated responses we considered the frequency response due to the interaction of the small volume, $d V$ of terrain with transmit and receive antenna. In this way we determined the received power versus frequency $(1-10 \mathrm{GHz})$ as well as the phase and amplitude for each frequency.

To identify effects of sensor geometry on distributed targets at further distances $(r>20 \mathrm{~m})$, a scatterometer requires small angular resolution. This often achieved with a smallbeamwidth antenna necessitating an increase in antenna aperture size. Also different antennas may essential for specific frequencies [17]. We avoided this need by careful selection of $d_{\mathrm{F}}$ and use of the Tripod mounts to alter $\theta_{i}$ in elevation.

\section{ANALYSIS OF AVERAGE REFLECTIVITY}

From our dielectric measurements of 10 and $20 \mathrm{wt}$. \% of MUT A and B we observed the $\varepsilon_{\mathrm{r}}{ }^{\prime}$ of oil sands falls between the dry LFS and beach sand with $10 w t . \%$ water content respectively (Fig. 5). Measured real permittivity values for beach sand and LFS agree with literature [1]. An important observation relevant to this work is a dielectric resonance effect observed for oil sands (both hard and viscous see MUT E - F Fig.2) in the upper C-band region ( $7-8 \mathrm{GHz})$. We believe the resonance seen in the permittivity of oil sand is due to the heterogeneous nature of oil sand. The presence of bitumen counteracts the presence of moisture in the $6.5-7.5 \mathrm{GHz}$ region. Both results have already been reported separately in [9] and [10] although we combined the results here to better understand the subsequent modeling and measurement results. 


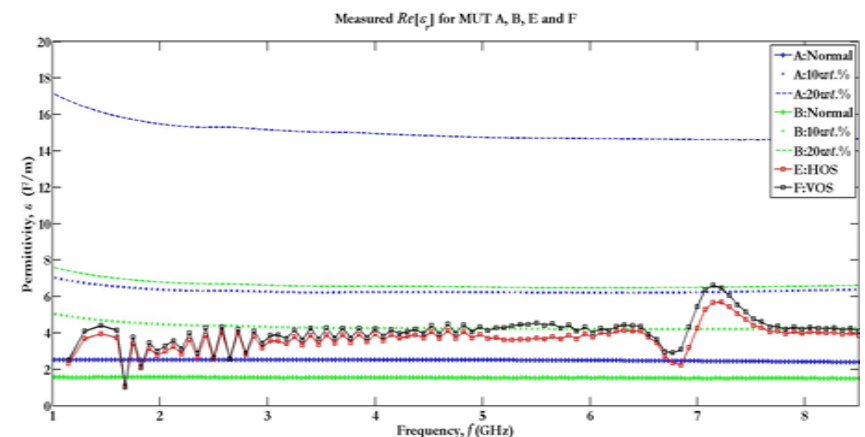

Fig. 5. Comparison of measured real dielectric permittivity, $\varepsilon_{r}^{\prime}$ for MUT A, $\mathrm{B}, \mathrm{E}$ and $\mathrm{F}$ at $f=1-8.5 \mathrm{GHz}$. MUT $\mathrm{A}$ and $\mathrm{B}$ results are presented for normal condition, 10 and $20 w t . \%$ water content.

The negative values from the dielectric permittivity measurements were smoothed out. Also unlike measurement results from MUT A and B the VOS results used as $\boldsymbol{d}_{\varepsilon}$, from (5) could not fit in to a $1^{\text {st }}$ or $2^{\text {nd }}$ order dispersion model for FIT. Therefore the raw dispersion values $\left(\mathrm{N}^{\text {th }}\right.$ order model) were used in the 3D EM models. Next we consider the (a) monostatic reflectivity for all the modelled terrain types (b) effect of surface roughness (c) effect of dielectric permittivity and finally (d) highlight the average reflectivity values from the modelled results of MUT A, C, D and E with the LSS.

\section{A. General Terrain Response}

The power received by the antenna due to the presence of terrain in the monostatic configuration is seen from the S11 results showing MUT A, C, D and E with the LSS model in $\mathrm{HH} / \mathrm{VV}$ mode shown in Fig. 6.

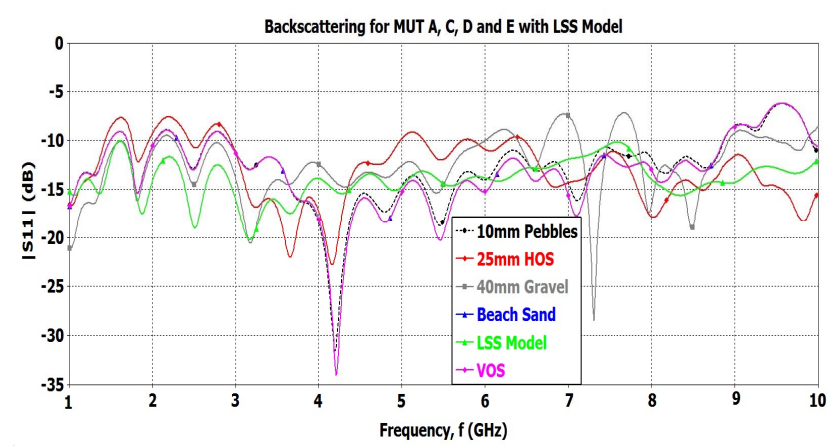

(a)

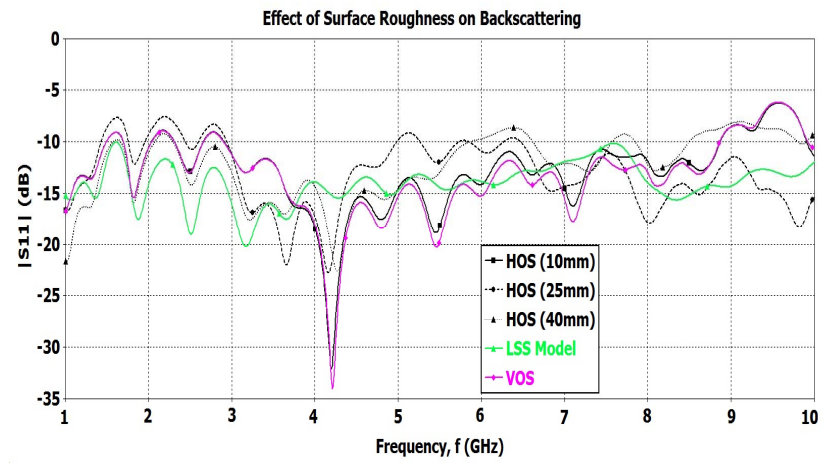

(b)

Fig. 6. Modelling results for LSS in HH polarisation (a) General terrain response for MUT A, C, D and E (b) Effect of surface roughness for VOS and three types of HOS.
A few key trends emerged. At low frequencies $(1-3 \mathrm{GHz})$ there is little variation across the terrain types due to the small sample size compared to wavelength $(d V<<\lambda)$. At higher frequencies homogenous barefaced terrain such as gravel and pebble produce $6 \mathrm{~dB}$ more scattered power compared to the LSS model alone in Fig.6a. At 9.5GHz the presence of bitumen in HOS reduces scattering produced by up to $4 \mathrm{~dB}$.

\section{B. Surface Roughness}

The effect of surface roughness for a realistic scenario was determined by considering three different roughness of HOS terrain with particle diameters of $10 \mathrm{~cm}, 25 \mathrm{~cm}$ and $40 \mathrm{~cm}$ and also VOS which has a relatively smooth surface (Fig. 2f). For similar material dielectric permittivity in the case of HOS, the S11 results show a $3 \mathrm{~dB}$ difference for every $15 \mathrm{~cm}$ increase of surface roughness at $7 \mathrm{GHz}$. This increases to $8 \mathrm{~dB}$ at higher frequencies (Fig. 6b). At $7 \mathrm{GHz}$ VOS yields the least scattering meaning that VOS will appear darker in radar imagery. This effect of bitumen presence leading to absorption of EM energy has also been observed at optical wavelengths [18] and [19].

\section{Dielectric Permittivity}

The effect of dielectric permittivity on scattering for MUT A-E was investigated using plane wave incident at $\theta_{\mathrm{i}}=90^{\circ}$ and E-field probes placed on the $0.1 \mathrm{~m} \times 0.32 \mathrm{~m}$ surface plane $(-z)$. MUT A-E was grouped in to three classes with similar surface roughness but different dielectric properties. At L-, C- and Xband terrain scattering mostly increased with frequency (Fig.7).

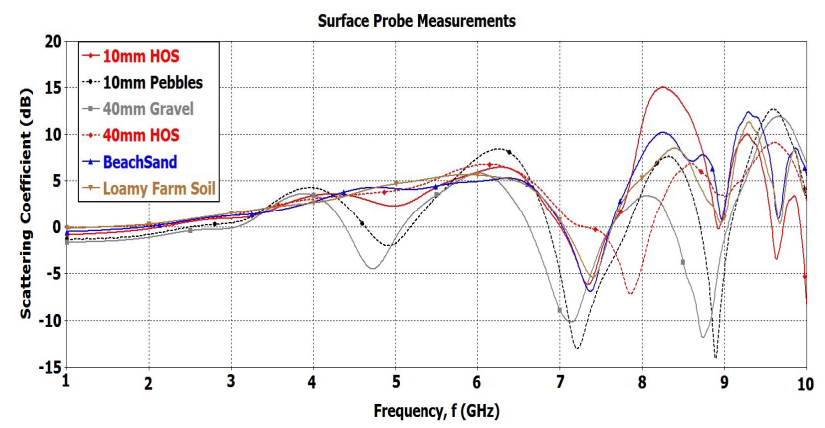

Fig. 7. Surface probe measurements of scattering coefficient for plane wave incident at $\theta_{\mathrm{i}}=90^{\circ}$

As already noted, HOS has greater dielectric permittivity variation requiring an $\mathrm{N}^{\text {th }}$ order model dispersion fit but constant $\varepsilon_{\mathrm{r}}{ }^{\prime}=3.05$ which is lower than quartz $\left(\varepsilon_{\mathrm{r}}{ }^{\prime}=3.7\right)$. Furthermore quartz is the main component in pebbles and gravel therefore HOS reflects more EM energy than both especially at L-, and X-band respectively. Similarly the proximity in real permittivity for beach sand and LFS produced a similar scattering response.

\section{Modelling and LSS Results}

The maximum and minimum reflectivity values of $\sigma^{0}$ in $\mathrm{dB}$ for MUT A - F was obtained using (11). Results for the six terrain types indicative of low backscatter environment are presented in table II. The average reflectivity value was obtained by post processing the E-field observed at the rear of the terrain $(+z)$ direction. In $\mathrm{HH}$ polarization mode the 
reflectivity from terrain at $10 \lambda$ distance was the same on both the front and rear terrain planes due to the relatively short distance $(0.32 \mathrm{~m})$ of the samples. This was also observed for VV polarization. However the better alignment of the LSS antenna plane with the terrain in the VV configuration resulted in higher backscattering (table II). This indicates the importance of imaging geometry in processing radar imagery.

There was good agreement between the measured and modeled results. It is believed that non-varying errors in the return signal were minimized by the SOLT calibration while environmental scattering effects from other objects were limited by the anechoic chamber which is EM silent. Comparison of results for $\mathrm{HH}$ and $\mathrm{VV}$ polarizations at $1 \mathrm{GHz}$, $7 \mathrm{GHz}$ and $10 \mathrm{GHz}$ showed strong correlation at low frequencies. However at X-band LSS geometry could account for the difference between empirical and modeled results.

TABLE II. AVERAGE REFLECTIVITY VALUES

\begin{tabular}{|l|c|c|c|c|c|c|}
\hline \multirow{2}{*}{ Surface } & \multicolumn{3}{|c|}{$\boldsymbol{\sigma}_{\boldsymbol{H} \boldsymbol{H}}$} & \multicolumn{3}{c|}{$\boldsymbol{\sigma}_{\boldsymbol{V} \boldsymbol{~}}^{\mathbf{0}}$} \\
\cline { 2 - 7 } & $\mathbf{1 ~ G H z}$ & $\mathbf{7 ~ G H z}$ & $\mathbf{1 0} \mathbf{~ G H z}$ & $\mathbf{1 ~ G H z}$ & $\mathbf{7 ~ G H z}$ & $\mathbf{1 0 ~ G H z}$ \\
\hline Pebbles & 5.004 & 1.006 & 1.854 & 27.23 & 2.854 & 5.070 \\
\hline Gravel & 5.284 & 0.850 & 1.564 & 26.33 & 3.120 & 5.670 \\
\hline Beach Sand & 0.618 & 0.048 & 0.067 & 2.973 & 0.218 & 0.343 \\
\hline LFS & 6.083 & 0.450 & 0.616 & 32.64 & 2.254 & 3.970 \\
\hline HOS & 5.912 & 0.525 & 0.796 & 35.28 & 2.014 & 3.622 \\
\hline VOS & 6.682 & 0.587 & 0.864 & 28.41 & 2.067 & 3.233 \\
\hline
\end{tabular}

\section{CONCLUSION}

The modeled and measured scattering results had a very strong correlation at $1 \mathrm{GHz}$. The capability of VNA based radar deployed as a laboratory scatterometer system has been proven. The presented configuration can be applied to emergent terrain backscatter measurements in situations where there is no access to airborne or spaceborne data such as oil sand exploration. The measurement results compared favorably with 3D EM models developed with FIT. Six datasets were modeled but four were measured at horizontal and vertical polarization covering monostatic angles between $0^{\circ}$ and $30^{\circ}$. Empirical datasets were recorded in an anechoic chamber. It was observed that for all angles the planar and corner reflectors provided the greatest reflectivity.

It is believed that the relatively small amount of terrain compared to wavelength $(\lambda=30 \mathrm{~cm}$ at $1 \mathrm{GHz})$ caused the imperceptible variation at low frequencies. However correcting for area produced more substantial reflection. Interestingly the effect of dielectric resonance of oil sands caused lower reflection in the upper $\mathrm{C}$-band region as against expectations. This could be due to the effect of Bitumen.

\section{REFERENCES}

[1] F.T. Ulaby, R.K. Moore and A.K. Fung, Microwave Remote Sensing Active and Passive, vol.2, Radar remote sensing and surface scattering and emission theory, Addison-Wesley Pub. Co, 1982, pp.457-1047.
[2] J. Berry and G. Prost, "Hydrocarbon exploration" in Remote Sensing for the Earth Sciences-Manual of Remote Sensing, $3^{\text {rd }}$ ed., A. Rencz, Ed. John Wiley and Sons, 1999, pp. 449 - 508.

[3] S.R. Cloude and E. Pottier, "An entropy based classification scheme for land applications of polarimetric SAR", IEEE Transactions on Geoscience and Remote Sensing, vol. 35, No. 1, Jan 1997, pp. 68-78.

[4] S.R. Cloude and E. Pottier, "A review of target decomposition theorems in radar polarimetry", IEEE Transactions on Geoscience and Remote Sensing, vol. 34, No. 2, March 1996, pp. 498-518.

[5] G.G. Schaber, "SAR studies in the Yuma Desert, Arizona: sand penetration, geology and the detection of military ordnance debris," Remote Sensing of the Environment, vol. 67, 1999, pp.320-347.

[6] F.T. Ulaby, "SAR biophysical retrievals: lessons learned and challenges to overcome," Proceedings of the $2^{\text {nd }}$ International Workshop on Retrieval of Bio- and Geo-physical Parameters from SAR Data for Land Applications, 21-23 October 1998.

[7] M.C. Dobson, F.T. Ulaby and L.E. Pierce, "Land cover classification and estimation of terrain attributes using synthetic aperture radar," Remote Sensing of Environment, vol. 51, 1995, pp. 199-214.

[8] J.R.Huyen, "Phenomenological theory of radar targets," $\mathrm{PhD}$ Dissertation, Technical University, Delft, The Netherlands, 1970.

[9] M. Ezeoke and K. Tong, "Modeling electromagnetic reflectivity of Agbabu oil sands from hyperspectral infrared reflectance spectra and dielectric properties at L-, C- and X-band frequencies”, Proceedings of the IEEE 5th Computational Intelligence, Communication Systems and Networks (CICSyN2013), 5-8 June, Madrid, Spain, 2013, pp.125-130.

[10] M. Ezeoke, K. Tong, A. Amiri and A. Al-Armaghany, "Effects of electrical and physical properties of barefaced terrain on backscatter response," Proceedings of the International Conference on Adances in Computing Electronics and Communication, (ACEC 2013), 12-13 October, Zurich, 2013, pp.89-93.

[11] M. Ezeoke, K. Tong, S. Shi, "A practical approach for modelling the electromagnetic radar signature of barefaced terrain for remote sensing", WIT Journal of Computational Methods Experiments and Measurements, vol. 190, 2014, in press.

[12] CST Microwave Studio: HF design and analysis Tutorial. In CSTComputer Simulation Technology, 2006.

[13] M. Clemens and T. Weiland, "Discrete electromagnetism with the finite integration technique", Progress in Electromagnetics Research, PIER 32, pp. 65-87.

[14] H. Schantz, "The art and science of ultrawideband antennas", The Artech House antenna and propagation library, Artech House, Incorporated, 2005, pp.248-257.

[15] L. Foged, A. Giacomini, L. Duchesne, E. Leroux, L. Sassi, J. Mollet, "Advanced modelling and measurement of wideband horn antenna", Proceedings of the $11^{\text {th }}$ International Symposium on Antenna Technology and Applied Electromagnetics (ANTEM 2005), 15-17 June, Saint-Main, France, 2005

[16] M.W. Whitt, F.T. Ulaby and K. Sarabandi, "Polarimetric scatterometer systems and measurements," in Radar polarimetry for geoscience applications, F.T. Ulaby and C. Elachi, Eds. Norwood, MA: Artech House, 1990, pp. 191-272.

[17] F.T. Ulaby, T.F. Haddock, J.R. East and M.W. Whitt, "A millimeterwave network analyzer based scatterometer", IEEE Transactions in Geoscience and Remote Sensing, vol. 26, No. 1, January 1988, pp. 75-81.

[18] E.A. Cloutis, M.J. Gafey and T.F.Moslow, "Characterization of minerals in oil sands by reflectance spectroscopy," Fuel, 74(6), 1995, pp.874-879.

[19] M. Ezeoke, K. Tong and K. Mubea, "Electromagnetic characterisation of terrain for unconventional petroleum exploration", Proceedings of the IEEE Radar Conference (RADARCON2014), 19-23 May, Cincinatti, USA, 2014, pp.990-995. 